\title{
Transracial Identity and the Effect on the Life Circumstances: A Pilot Study in 14 Participants
}

\author{
Franziska Sülter, Erich Kasten \\ Medical School Hamburg (MSH), Department of Human Sciences, Am Kaiserkai 1, Hamburg, Germany \\ Email: franziska.suelter@gmx.de,EriKasten@aol.com
}

How to cite this paper: Sülter, F., \& Kasten, E. (2021). Transracial Identity and the Effect on the Life Circumstances: A Pilot Study in 14 Participants. Open Journal of Social Sciences, 9, 471-479.

https://doi.org/10.4236/jss.2021.97034

Received: May 24, 2021

Accepted: July 25, 2021

Published: July 28, 2021

Copyright $\odot 2021$ by author(s) and Scientific Research Publishing Inc. This work is licensed under the Creative Commons Attribution International License (CC BY 4.0).

http://creativecommons.org/licenses/by/4.0/

\begin{abstract}
Goal: The term "Transracial Identity" first appeared in science around 2017. The people concerned identify themselves with a different ethnicity or nationality, respectively they feel a strong attraction to it, in contrast to the one they were born into. Transracial identity can possibly be compared to Transgender or Body Integrity Dysphoria. The leading question of this article is: How does "transracial identity" affect the life circumstances of those affected? Methods: A questionnaire with items about personal information and the feelings of being transracial was created. The Big-Five-Inventory-10 was used aiming at investigating parallels of personality in Transracials. In addition, Düsseldorf's Questionnaire of Transidentity, and the Questionnaire of the Body Integrity Identity Disorder were used to find out parallels with Transgender or Body Integrity Dysphoria. Sample: The study consists of a sample of 14 subjects, who identify as transracial (average age $30 \pm 8.9$ years, 35.7\% $(\mathrm{n}=5)$ male and $64.3 \%(\mathrm{n}=9)$ female $)$. Participants from nearly all ethnicities identify with nearly any other race. Results: A correlation between discontent of life and the desire to change the race could not be stated. The hypothesis that individuals, identifying with another ethnicity or race, more often suffer from a mental disease than the general population, could be accepted. However, it remains unclear whether the dissatisfaction with one's own race can cause depression or if depression is the cause of the "transracial identity" and whether those affected believe they can solve their psychological problems by changing to a new identity. Parallels to Transgender and Body Integrity Dysphoria were found. Regarding attractivity, unhappiness with their appearance is of vital importance. Thus, transracials' satisfaction with their bodies and faces is rather low. This can be explained by the fact that their current biological ethnicity, with which their appearance is also connected, does not correspond to the desired ethnicity.
\end{abstract}




\section{Keywords}

Transracial, Transracial Identity, Identity, Ethnicity, Transidentity

\section{Introduction}

Belonging to an ethnic race is genetically determined and therefore includes an abundance of typical characteristics, in particular skin- and hair-color, body structure, and facial features. People concerned do consider their ethnicity-being their fate; they were born with these characteristics and grew into their group. Partly there is dissatisfaction, for example in dark-skinned people who were born into a world in which light-skinned people have the power. Belonging to a race or nationality has historically played an important role in the history of mankind, not infrequently there were wars or oppression due to different skin colors, up to the extermination of whole tribes (Osterhammel, 1995). Today, the world is growing together, skin colors and nationalities are mixing more and more, within the same state in the 21st century almost all ethnic types occur. However, it seems to be a fallacy to believe that belonging to a skin colour or ethnic culture no longer plays a role. People still define themselves by this and even today there is a stigmatization of members of other cultures. The very existence of transracials shows how important belonging to a race contributes to finding identity. The feeling of belonging to the "wrong" ethnicity can trigger feelings of inferiority and psychological crises. It therefore seems important not to underestimate this fact in our social life even in the mixed cultures of the modern world. Movements such as "Black is beautiful" try to compensate this arousing pride in the given ethnicity. But there are people who feel that their ethnicity is wrong. They were born with the typical characteristics of one ethnic group, but this appearance does not correspond to their mental feeling. Past studies used the term "transracial" to describe individuals who identify with or feel a strong attraction to another ethnicity, as opposed to the current one into which they were born (Tuvel, 2017). This identification goes beyond mere wishful thinking or a sense of belonging to a particular group. The term identity describes "the unique combination of personal, distinctive data of an individual" (Seiffge-Krenke, 2012). This data includes, among other things, ethnicity. Identity develops accordingly in interplay of "belonging" and "separating oneself" (Ermann, 2011). Thus, as the origin of "transracial identity", one could assume a "belonging" and "setting oneself apart" to or from a certain cultural group. Thus, the term "transracial" can be understood as beyond, that is, beyond the biological, original identity and associated ethnicity.

The main focus of this study is an open discourse about this unexplored topic, so it can raise awareness and understanding for "transracials" and another way of living (e.g. Personal identity).

Transracial can possibly be compared to Transgender, i.e. people living in a 
body with the genital organs not suitable to the mental picture of their gender. Another related disorder is Body Integrity Dysphoria (former: Body Integrity Identity Disorder). These are individuals having a healthy body but longing for a disability, e.g. an amputation or paralysis. This group also has the intense feeling of living in the wrong body; their brain is that of a physically disabled person, but the body is intact.

The guiding/key question of this article is: "What influence does the 'transracial identity' have on the life circumstances of the persons concerned?"

\section{Methods}

An online-questionnaire (Unipark, Questback) with items about personal information and the feeling of being transracial; e.g. dissatisfaction with the innate ethnicity, strength of the desire to change the race, extend of the changes in life due to change in ethnicity (each on a 5 step-scale) was developed, In addition, life satisfaction was asked on a bipolar 5-step scale. Finding out about parallels in personality of Transracials, the Big-Five-Inventory-10 (BFI-10) was performed (Rammstedt, Kemper, Klein, Beierlein, \& Kovaleva, 2012). The "Big Five" are Openness, Conscientiousness, Extraversion, Agreeableness and Neuroticism. In addition, some adapted questions from the Düsseldorf's Questionnaire of Transidentity (Söder, 1998), and the Questionnaire of the Body Integrity Disorder-Screening of severity and intensity (Fischer, Schnell, \& Kasten, 2015) were used to analyse parallels of Transracials with Transgender or Body Integrity Dysphoria. All participants were contacted via Social Media.

\section{Sample}

The study consists of a sample of 14 participants, identifying themselves as transracial. The representativeness of the data is limited because of the small sample. At this point the representativeness is not decisive because the main focus of this pilot study is to highlight the existence of the "transracial identity" and the circumstances leading to it, as well as the impact on the life circumstances. The average age of the participants was $30(S D=8.97)$ years $(\min 19 \mathrm{y}$., $\max 46$ y.). The sample included $35.7 \%$ male $(n=5)$ and $64.3 \%$ female participants $(n=9)$. Table 1 shows the innate ethnicity and the race they identify with. It has to be mentioned that participants from nearly all ethnicities identify themselves with nearly all other races. Regarding sexual orientation $42.9 \%(\mathrm{n}=$ 6) of participants were heterosexual, 35.7\% $(n=5)$ bisexual, and $21.4 \%(n=3)$ homosexual.

Table 1 presents the innate and identified ethnicities. The participants $(\mathrm{n}=$ 6), who stated identifying with a Caucasian ethnicity provide the highest frequency; a smaller amount of participants identified themselves with Caribbeans, Jamaicans and Pacific Islanders $(\mathrm{n}=3)$, two identified with an African and two with a Latin-American ethnicity, one participant had the desire to have Asian features. 
Table 1. Description of the participants with the born-in and identified nationality or ethnicity.

\begin{tabular}{cccc}
\hline & innate Ethnicity & Living in & Identified ethnicity \\
\hline $\mathbf{1}$ & Caucasian & North-America & African \\
$\mathbf{3}$ & Caucasian & North-America & Latin-amof terican \\
$\mathbf{4}$ & Eastern European & North-America & Caucasian \\
$\mathbf{5}$ & Eastern European & Europe (Croatia) & Caucasian (British or US-American) \\
$\mathbf{6}$ & Caucasian & North-America & Caucasian but typically Scandinavian \\
$\mathbf{7}$ & German & Europe (Germany) & Latin-American \\
$\mathbf{8}$ & Asian & Asia & Caucasian \\
$\mathbf{9}$ & Chilean & South-America & Caucasian (but typically British) \\
$\mathbf{1 0}$ & Brazilian (dark skinned) & South America & Caucasian German or eastern European) \\
$\mathbf{1 1}$ & African & North-America & Asian \\
$\mathbf{1 2}$ & Caucasian & North-America & Carribean/Jamaican (dark skinned) \\
\hline $\mathbf{1 3}$ & African & North-America & Pacific Islander \\
\hline
\end{tabular}

\section{Results}

Looking at the personality of the Transracials, Table 2 presents an overview of the results.

The average value of the question "How strong is the wish to be accepted as another ethnicity?" ( 1 = very weak to $5=$ very strong) was $3.64(\mathrm{SD}=1.78)$.

The strength of the desire to belong to another ethnicity on this 5-step scale (1 $=$ strong negative impact on the wellbeing, $3=$ average, and $5=$ positive impact on the well being) resulted in an average value of $1.86(\mathrm{SD}=1.29)$.

The first cluster of hypotheses investigated life satisfaction of Transracials. A significant correlation between the extent of dissatisfaction and the innate ethnicity and life satisfaction, in Spearman's $\mathrm{R}$ couldn't be found $(\mathrm{r}=0.022, p=$ $0.94)$. Also, the results for the hypothesis of a correlation between the life-satisfaction and the intensity of the desire being a part of another ethnicity missed statistical significance. The hypothesis regarding the connection between life satisfaction and the change of life circumstances showed a negative insignificant correlation. Even if the participants could achieve to be a part of the desired ethnicity $(\mathrm{r}=$ $-0.430, p=0.125)$. The "transracials" estimated their general life satisfaction with an average score of $2.43 \mathrm{SD}=1.22$, on a scale between $1=$ very unhappy to 5 = very happy as rather unhappy.

In the question: "How much would your circumstances of life differ from today, if you had the chance to be a part of this racel ethnicity?" the participants achieved an average result of $4.64,(\mathrm{SD}=0.75)$ on the above mentioned 1 to 5 scale. 
Table 2. Results of the Big-Five Inventory (BFI-10) in 14 participants with Transracial Identity on a 5 -step-scale (average $=3.0$ ).

\begin{tabular}{cc}
\hline Personality Dimension & Result \pm SD \\
\hline Neuroticism & $3.79 \pm 1.01$ \\
Extraversion & $3.39 \pm 1.38$ \\
Openness & $4.10 \pm 0.90$ \\
Compatibility & $3.36 \pm 0.95$ \\
Conscientiousness & $3.64 \pm 0.40$ \\
\hline
\end{tabular}

The results of the questions: "What kind of feelings and emotions do you experience when you see or meet a person of the ethnicity you identify with?" and "What feelings do you have when you see people of your innate ethnicity?" are shown in Table 3.

Transgender has a twisted gender identification, and a disproportionately large number of homosexuals and transgender can be found in those affected by Body Integrity Dysphoria. In the next hypothesis the sexual orientation of the Transracials was analyzed. Their sexual orientation was compared to the demographic frequency based on statistics. A study from the National Health Statistics Reports stated that $96.9 \%$ of all adults are heterosexual, $1.6 \%$ homosexual and $0.7 \%$ bisexual (National Health Statistics Reports, 2013). In the small sample of the transracial group, $42.86 \%$ were heterosexual, $21.43 \%$ homosexual and $35.71 \%$ identified themselves as bisexual. Those numbers differ drastically from the normal demographic frequency.

Ten individuals (71.4\%) reported body modifications to more closely match their identified ethnicity. For example: These included altering or adapting their hairstyle and color to appear more African American or Latino. In contrast, others reported bleaching or lighting their hair to more closely resemble the so-called Caucasian type. Several participants used certain kinds of make-up or clothing style. Several transracials stated that they tanned their body to get a darker skin color. One person mentioned that he changed his name into an African sounding. Another person stated that he had undergone a nose job to look more British. In addition, this person colored his hair and wears blue contact-lenses. He stated that he had undergone a cheek fat removal in order to look more British. One participant stated that to the best of his knowledge, it was not possible to make a full transition to another ethnicity, in the aforementioned case from Latino to White, or Caucasian. Therefore, he grew a beard, to make his lips look thinner and used a lot of sunscreen to make his skin look lighter and not darker in the future. Another participant had lip injections done and had ethnic tattoos, as well as a tanned skin to look more like the desired ethnicity. Finally, someone stated that she regularly wears long, wavy wigs and darker make-up, to make her yellowish looking skin appear tanned. A female person stated that, in addition to her clothing, she changed her general appearance and personality to match her desired Asian ethnicity. 
Table 3. Results on questions of how transracials feel when they see a person of the desired ethnic group or their own ethnic group ( 5 step scale, average $=3.0$ ).

\begin{tabular}{ccc}
\hline & Desired ethnicity & Innate ethnicity \\
\hline calm $-->$ excited & $3.64 \mathrm{SD}=1.65$ & $2.50 \mathrm{SD}=1.79$ \\
sad $-->$ happy & $3.57 \mathrm{SD}=1.70$ & $2.50 \mathrm{SD}=1.65$ \\
aggressive $-->$ peaceful & $2.86 \mathrm{SD}=1.61$ & $2.29 \mathrm{SD}=1.44$ \\
\hline
\end{tabular}

Nine participants (64.3\%) planned to change their appearance by "soft" methods such as wearing. ethnic-typical clothes, tanning or lighting their skin (e.g. by totally avoiding the sun), or changing their hairstyle. Others planned to undergo plastic surgery; to let their lips appear more or less voluminous. One participant stated that he wanted a surgery on eye, lip and nose in order to match his Asian appearance to the Caucasian type. A Chilean participant, who identified as British, planned to have a surgery on the chin. In addition, he would like to have a permanent change in eye color through a surgical procedure with implants. This method was frequently mentioned. Another person stated that he would do whatever it took to outwardly conform to their "desired ethnicity."

In addition, the participants were asked whether they had suffered from a mental disorder. 11 participants (78.6\%) indicated that they had., $\mathrm{n}=10$ depression (one suffered from a manic depression); $\mathrm{n}=9$ anxiety disorder (one with panic attacks); $\mathrm{n}=4$ posttraumatic stress disorder (PTSD), $\mathrm{n}=1$ schizophrenia, $\mathrm{n}=1$ social behavior disorder; $\mathrm{n}=1$ obsessive-compulsive disorder, $\mathrm{n}=1$ Asperger's syndrom, $\mathrm{n}=1$ borderline personality disorder; and $\mathrm{n}=1$ learning disorder. Compared to the general population only $27.8 \%$ of the adult population in Germany suffer from a mental illness (DGPPN, 2019).

Transgender people or those suffering from Body Integrity Dysphoria experience their difference for the first time in childhood. In order to find out if transracials also notice their being different in that early age a suitable question was generated. $30.8 \%(n=4)$ of the transracials reported noticing this in the childhood before an age of 10 years; $53.9 \%(n=7)$ aged 10 - 15 years; two individuals $(15.4 \%)$ in adulthood, i.e. 25 and 30 years (see Figure 1). Therefore, the hypothesis that transracial identity develops in early age in most cases, could be confirmed.

Another aspect that was asked in the questionnaire was the age of the first contact with a person of the desired ethnicity/race One person indicated having an adopted sibling who matched the identified ethnicity. Another participant stated that he watched German, British, and American television as a child and felt a strong attraction to the people depicted, as opposed to people in his environment. One person indicated that a majority of childhood friends belonged to the ethnic group later identified.

What reasons were given? $71.4 \%$ of the participants $(n=10)$ named reasons: Firstly, it was stated that as a child the idea of being a different person living in a different environment was used as one of the only coping mechanisms to 


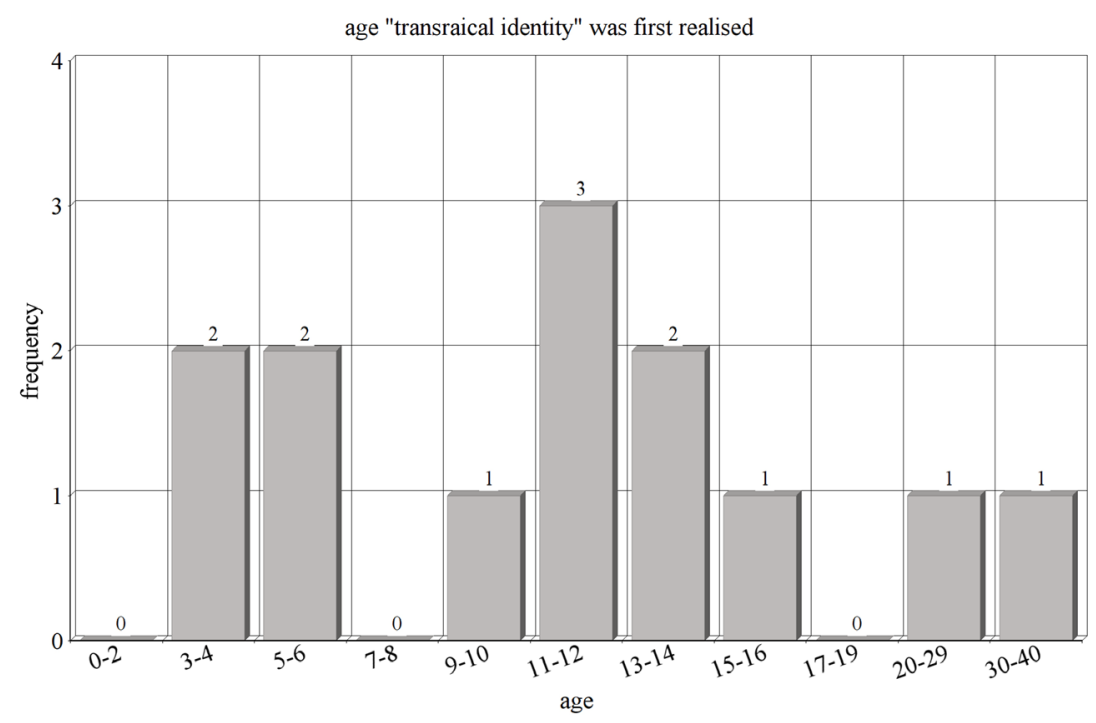

Figure 1. Frequency distribution: age, in which the "transracial identity" was noticed first.

mentally and emotionally escape from the violent family environment. Another participant wrote that the cultural values and philosophies of the identified ethnicity were in line with their own attitudes. One participant said that he disliked the culture and the country he was from, had never felt he belonged, had experienced bullying, and the participant generally could not integrate into his environment. In the future, he would want to start a new life, as a potentially happy person (of another race).

One (Asian) person stated not being able to identify with the Asian-stereotype, likewise, the culture had no connection to his own personality. One participant wrote, he feels "off-color" with the innate ethnicity. One person grew up in the environment of the identified ethnicity and therefore felt a full identification with that ethnicity and not the biological ethnicity another participant said. that visually and from their physique, as well as from their personality and behavior, they more closely resembled to the expected image of another ethnicity. One reason given for the existing "transracial identity" is the affection for the culture and related music and also that the culture seemed familiar and natural to them.

Finally, one participant growing up in a multicultural environment and thus having relatives and friends from different cultures influenced his development.

As exiting role models in childhood $\mathrm{n}=4$ participants named relatives with a different ethnicity. One participant stated his teacher being his role model. Another person named Rachel Dolezal (a well known transracial) as a role model. Famous personalities were also named as role models.

Our study also asked, whether transracials have a low body satisfaction. On the scale $1=$ too thin, $2=$ thin, $3=$ normal, $4=$ fat and $5=$ too fat, the average value, describing the body satisfaction, was $3.7(\mathrm{SD}=1.6)$, i.e. the "transracials" perceive themselves as normal to fat. Satisfaction with the face was questioned on the scale $1=$ very dissatisfied, $2=$ dissatisfied, $3=$ normal, $4=$ pleased, $5=$ 
very pleased. With an average value of $2.3(\mathrm{SD}=1.3)$ transracials tended to be slightly dissatisfied with their faces. On average, the participants classified themselves as a little too fat and normal to slightly not attractive.

\section{Discussion}

\subsection{Reasons behind the "Transracial Identity"}

Nease (Nease, 2017) underlines the connection between asexuality and homosexuality with the "transracial identity" in her study about the "transracial identity", similar findings could be recorded in this study. Bisexuality and homosexuality occur above-average frequency among "transracials". Whether these findings are due to chance or whether the "transracial identity" is positively correlated to bisexuality and homosexuality is not clear and needs to be recorded in future studies. Regarding the personality of the "transracials", the dimension openness is the most pronounced. This indicates that individuals who identify with a different ethnicity/race are more open to new experiences and are equally likely to try new things. This could be related to the fact that the test persons are open to surgical procedures and the whole phenomena of "transracial identity" is something so innovative and new that people who intensively dealt with this issue to the point to take part in this study, have a high degree of openness in personality. The test persons stated that they felt happy when they saw a person of the desired ethnicity. This could be interpreted as an emotional causality of "transracial identity," which is further emphasized by the reasons given by the test persons.

\subsection{Answering the Leading Question}

In contrast to our assumption of a correlation between the level of dissatisfaction with the innate race, the level of life discontent, and the intensity of the desire belonging to a different ethnicity, these variables had no significant influence on the desire to change the race. Transracial identity does not have a negative impact on life circumstances, especially life satisfaction. The hypothesis that individuals, who identify with another ethnicity or race, more often suffer from mental disorders than the general population could be accepted. This leads to the question whether the "transracial identity" and the desire to belong to another ethnicity, or the dissatisfaction with one's own ethnicity, trigger mental disorders or whether the "transracial identity" is a symptom of an existing mental disorder or even a mental disorder in its own right. Both are likely true, as evidenced by participants' testimony.

Regarding the age, the first symptoms appear parallel to Transgender and Body Integrity Dysphoria which could be found. Most participants stated that they noticed their identification with another ethnicity aged 10 to 15 for the first time. Considering attractivity, unhappiness with their appearance is of vital importance. Thus, body satisfaction, with the body in general and the face in particular, turns out to be rather low among the "transracials". This can be ex- 
plained by the fact that their current biological ethnicity, with which their appearance is also connected, does not correspond to the desired ethnicity.

\section{Acknowledgements}

We thank all participants of this study who spend their time with our questionnaire.

\section{Annotation}

Every description of the race or skin color is non-judgmental.

\section{Conflicts of Interest}

The authors declare no conflicts of interest regarding the publication of this paper.

\section{References}

Ermann, M. (2011). Identität, Identitätsdiffusion, Identitätsstörung. Psychotherapeut, 56, 135-141. https://doi.org/10.1007/s00278-011-0813-8

Fischer, M., Schnell, T., \& Kasten, E. (2015). Body Integrity and Identity Disorder: Development and Evaluation of an Assessment of the Severity. American Journal of Applied Psychology, 4, 76-82. https://doi.org/10.11648/j.ajap.20150403.15

National Health Statistics Reports (2013).

Nease, C. (2017). Transracial Identity. Dissertation. School of Professional Studies, Gonzaga University.

Osterhammel, J. (1995). Kolonialismus: Geschichte, Formen, Folgen. Verlag C. H. Beck.

Rammstedt, B., Kemper, C., Klein, M., Beierlein, C., \& Kovaleva, A. (2012). Eine kurze Skala zur Messung der fünf Dimensionen der Persönlichkeit: Big-Five-Inventory-10 (BFI-10), Testmanual, Leibniz Institut für Sozialwissenschaften.

Seiffge-Krenke, I. (2012). Therapieziel Identität Veränderte Beziehungen, Krankheitsbilder und Therapie. Klett-Cotta.

Söder, M. (1998). Düsseldorfer Fragebogen zur Transidentität, test manual. HeinrichHeine-Universität.

Tuvel, R. (2017). In Defense of Transracialism. Hypatia, 32, 263-264.

https://doi.org/10.1111/hypa.12327 